\title{
First International Workshop on Multi Product Line Engineering (MultiPLE 2013)
}

\author{
Leon Moonen \\ Simula Research Laboratory \\ Oslo, Norway \\ leon.moonen@computer.org \\ Mithun Acharya \\ ABB Corporate Research \\ Raleigh, USA \\ acharyamithun@gmail.com
}

\author{
Razieh Behjati \\ Simula Research Laboratory \\ Oslo, Norway \\ raziehb@simula.no
}

Bedir Tekinerdogan
Bilkent University
Ankara, Turkey
bedir@cs.bilkent.edu.tr

\author{
Rick Rabiser \\ Johannes Kepler University \\ Linz, Austria \\ rick.rabiser@jku.at
}

Kyo-Chul Kang
POSTECH
Kohang, Korea
kck@postech.ac.kr

\begin{abstract}
In an industrial context, software systems are rarely developed by a single organization. For software product lines, this means that various organizations collaborate to provide and integrate the assets used in a product line. It is not uncommon that these assets themselves are built as product lines, a practice which is referred to as multi product lines. This cross-organizational distribution of reusable assets leads to numerous challenges, such as inconsistent configuration, costly and time-consuming integration, diverging evolution speed and direction, and inadequate testing.

The MultiPLE workshop is aimed at discussing the challenges involved with the development and evolution of multi product lines and the assets used for their production.
\end{abstract}

\section{Categories and Subject Descriptors}

D.2.0 [Software Engineering]: General; D.2.13 [Software Engineering]: Reusable Software; D.2.9 [Software Engineering]: Management; K.6.3 [Management of computing and information systems]: Software Management

\section{Keywords}

Multi product lines, software ecosystems, cross-organizational product line engineering, design, evolution, certification.

\section{MOTIVATION AND TOPICS}

Software product line engineering is a paradigm for developing families of similar software products, through developing, maintaining and configuring reusable assets. In an industrial context, software systems are rarely developed by a single organization. For example, in the case of large-scale product families it is common practice that various suppliers

Permission to make digital or hard copies of part or all of this work for personal or classroom use is granted without fee provided that copies are not made or distributed for profit or commercial advantage and that copies bear this notice and the full citation on the first page. Copyrights for thirdparty components of this work must be honored. For all other uses, contact the Owner/Author.

Copyright is held by the owner/author(s).

SPLC 2013, Aug 26-30 2013, Tokyo, Japan

ACM 978-1-4503-1968-3/13/08.

http://dx.doi.org/10.1145/2491627.2499882 and integrators collaborate throughout the development life cycle to provide and integrate the required subsystems and components. These components and subsystems themselves are often built as product lines (or as parts of product lines) and they are generally managed independently of each other, having their own specific requirements and stakeholders. In the literature, such "composite" product lines are most commonly referred to as multi product lines [4].

Multi product lines differ from single product lines in the sense that their assets are distributed across various organizations. The cross-organizational distribution of the reusable assets introduces several challenges, particularly inconsistent configuration of components and development artifacts, costly and time-consuming integration processes, and inadequate testing [4]. In addition, many of the product lines involved in a multi product line evolve in their own direction and at their own speed, especially since it is not uncommon that they are suppliers for more than one multi product line. Consequently, constant care needs to be taken to maintain the integrity of each of these composites [2].

\subsection{Topics}

The MultiPLE workshop focuses on addressing the specific challenges involved with managing the development and evolution of multi product lines and the assets that are used for their production. This includes the challenges that are related to the coordination and collaboration of organizations that produce product lines which contribute in forming larger multi product lines. The topics of interest include, but are not limited to:

- scoping for multi product lines - domain requirements engineering for MPLs

- (cross-organizational) modeling of multi product lines - commonality and variability analysis

- MPL integration and interoperability management

- product derivation \& configuration management

- asset management

- transition strategies

- (cross-organizational) evolution of MPLs

- model maintenance

- change impact analysis

- metrics and measurement

- MPL certification, verification and validation

- industrial experiences \& empirical studies on MPLs 
- MPLs and software ecosystems

- MPLs in the open source world

- non-technical issues surrounding MPLs

- economic and legal issues

- organizational, managerial, and process issues

- MPL training for organizations

\section{GOALS AND EXPECTED RESULTS}

\subsection{Goals}

The main goal of the MultiPLE workshop is to identify and better understand the challenges that are involved with managing the engineering and evolution of multi product lines and the cross-organizational distribution of the assets that are used for their production.

We aim to reach this goal by bringing together practitioners and researchers from the (multi) product line engineering community with practitioners and researchers that have expertise on related engineering challenges, such as ultra-large-scale systems [5], software ecosystems [1], compositional product lines [2], and product populations [6].

\subsection{Expected Results}

We expect to gain a common understanding of the challenges and issues related to multi product line engineering, and identify potential and synergy among the aforementioned fields to address those challenges and issues. We aim to achieve this understanding by focusing on the following three outcomes:

1. characterize and model the identified challenges and issues in MPL engineering,

2. collect solutions and techniques from related fields and assess their potential to address the needs of multi product line engineering, and

3. propose a research agenda for managing cross-organizational development and evolution of multi product lines and the assets used for their production.

\section{WORKSHOP FORMAT}

The workshop is scheduled to take one day. In order to build a research agenda to tackle the previously mentioned challenges, the workshop will have a strong focus on discussion and interaction.

\subsection{Schedule}

The workshop starts with an interactive introduction session, which is followed by focused sessions of short presentations followed by a longer discussion. In addition, we will have three working sessions that are built around interactive discussion techniques such as collaborative affinity diagramming and fishbowl panel discussions. These sessions are respectively aimed at building a common understanding of the challenges, discussing directions for potential solutions and establishing a common research agenda. The workshop will close with a "wrap-up" session where we will summarize the results and identify open issues.

\subsection{Workshop Activities}

To address the first outcome, we will use a combination of affinity mapping and card sorting to collect and organize issues and challenges of multi product line engineering. Affinity mapping and card sorting are popular techniques for con- ceptualization and help to get a group of people to discuss and organize their collective knowledge [3]. Participants will be given index cards and are asked to write down issues and challenges, one per card. Next, participants will be asked to collectively organize the cards into piles that represent similar concepts. Participants are allowed to merge or redistribute piles and move single cards, and will be asked to come up with a single name to describe the challenge represented by each pile. A picture will be taken of each pile, and used to drive the research agenda discussions. The contents of the piles will also be archived on the workshop website.

To address the second outcome, the participants will discuss if and how solutions and techniques from related fields can potentially address the needs of multi product line engineering. In order to drive the discussion, the open "Fishbowl" technique will be used. This technique is a variant of a panel discussion, where the audience sits in concentric circles around a set of four or five chairs for the panelists in the innermost circle (the fishbowl). One of the panelist seats is initially kept empty. After the moderator introduces the topic, the panelists start discussing. The audience listens in on the discussion but does not participate. However any member of the audience can, at any time, join the fishbowl by taking the empty chair. When that occurs, one of the current panelists must leave the fishbowl so that there is again a free chair. This process repeats until the time runs out, after which the moderator summarizes the discussion.

To address the third outcome (the research agenda), we aim to have a plenary discussion that is driven by the various piles collected in the card sorting session.

\subsection{Dissemination of results}

We maintain a workshop website where we will collect material in preparation of the workshop, and archive the material produced and collected during the workshop for future reference. This website is available at:

https://sites.google.com/site/wMultiPLE2013/

Accepted papers will be published in the ACM Digital Library as part of the SPLC 2013 Workshop Proceedings.

The organizers aim to synthesize the results in a workshop report that discusses the challenges brought forward, the progress made on the roadmap, and the open issues that still need to be addressed. This report will be made available from the workshop website and will be submitted to a suitable venue.

\section{REFERENCES}

[1] J. Bosch. From software product lines to software ecosystems. In SPLC'09, 2009.

[2] J. Bosch. Toward compositional software product lines. IEEE Softw., 27(3), 2010.

[3] D. Gray, S. Brown, and J. Macanufo. Gamestorming: A Playbook for Innovators, Rulebreakers, and Changemakers. O'Reilly Media, 1 edition, July 2010.

[4] G. Holl, P. Grünbacher, and R. Rabiser. A systematic review and an expert survey on capabilities supporting multi product lines. Inf. Softw. Technol., 54(8), 2012.

[5] L. Northrop et al. Ultra-Large-Scale Systems. The Software Challenge of the Future. Carnegie Mellon University, 2006.

[6] R. C. van Ommering. Beyond product families: Building a product population? In IW-SAPF, 2000. 